\title{
Spectrophotometric assessment of the effectiveness of Opalescence PF 10\%: A 14-month clinical study
}

\author{
S.R. Grobler', R. Hayward, S. Wiese, M.H. Moola, T.J. van W Kotze \\ Oral and Dental Research Institute, Faculty of Dentistry, University of the Western Cape, \\ Tygerberg, South Africa
}

\begin{abstract}
Objectives: To evaluate the effectiveness of Opalescence PF 10\% just after treatment, at 6month follow-up and at a 14-month follow-up period. Methods: Opalescence PF 10\% was applied nightly for 14 days. The color of teeth 11 and 21 of 17 subjects were measured with a spectrophotometer $\left(\mathrm{L}^{*} ; \mathrm{a}^{*} ; \mathrm{b}^{*}\right)$ before treatment, just after treatment (14 days), after 6 months and after 14 months. Subjects were instructed to take note of any tooth sensitivity.

Results: For all three components $\left(\mathrm{L}^{*}, \mathrm{a}^{*}\right.$ and $\left.\mathrm{b}^{*}\right)$ statistical significant differences $(\mathrm{p}<0.05)$ in the values between base-line, after treatment (14 days later), after 6 months and after 14 months were found (Wilcoxon Signed Rank Sum Test). The decrease in $\mathrm{L}^{*}$ was about $20 \%$ after 6 months and about 50\% after 14 months. The a* value decreased approximately 14\% after 6 months but was worse after 14 months than at the beginning. The $b^{*}$ value decreased the least with about $9 \%$ after 6 months and about $8 \%$ after 14 months. The decrease in DE_ab was_20\% after 14 months. Less than $20 \%$ of the subjects experienced mild tooth sensitivity just after treatment.
\end{abstract}

Conclusion: Significantly whiter teeth were found after treatment as well as after a 6-month follow-up period. The whiteness/brightness $\left(\mathrm{L}^{*}\right)$ decreased with _50\% after 14 months and the $\mathrm{a}^{*}$ value with $\_50 \%$ after 7 months, while the yellowness ( $b^{*}$ value) remained even after 14 months.

Clinical implications: The product is an effective tooth whitener resulting in only low tooth sensitivity. Re-bleaching could be done at about 14 months.

\section{Introduction}

Tooth whitening in cosmetic dentistry has experienced an evolution in the last decade. The different ways in which teeth can be whitened include dentist-supervised home bleaching (nightguard vital bleaching), in-office or power bleaching, a combination of in-office and take-home bleaching as well as over-the-counter whitening products for use at home.

The mechanisms of tooth bleaching occur by the diffusion in and through enamel to cause oxidation and therefore lightening of organic pigments or chromogens, particularly within the

1 Corresponding author at: Oral and Dental Research Institute, Faculty of Dentistry, Private Bag X1, Tygerberg 7505, South Africa. Tel.: +27 21 937 3023; fax: +27 21937 3025. E-mail address: srgrobler@uwc.ac.za (S.R. Grobler). 
dentin. ${ }^{1,2}$ Lower concentrations of hydrogen peroxide of up to $10 \%$ or carbamide peroxide (10-22\%) are used for at-home bleaching and higher concentrations (30-35\%) are recommended for in-office procedures. ${ }^{3}$ However, $10 \%$ carbamide peroxide is still the most commonly used bleaching agent ${ }^{4-7}$ because of its reported safety and effectiveness. . $^{3,8-10}$

Whether bleaching of teeth results in temporary or permanent damage to enamel or dentine or whether even low concentrations of hydrogen peroxide (as used in night- guard bleaching) causes damage to enamel and to the pulp, is still controversial,. According to Pugh G et al. ${ }^{11}$ low concentrations of hydrogen peroxide $(3.5 \%, 7 \%$ and $12 \%)$ are not expected to adversely affect the enamel and pulpal enzymes. However, a recent study ${ }^{12}$ revealed that all four different peroxide concentrations (from 3.35 to $15 \% \mathrm{H}_{2} \mathrm{O}_{2}$ ) of Opales- cence products damaged enamel, while the most damage was done when teeth were treated for longer periods.

A 6-month follow-up study ${ }^{13}$ on patients that had bleached for $2 \mathrm{~h}$ /day with two different carbamide peroxide concentrations (10\% and $16 \%$ respectively) for 3 weeks, showed that the color of teeth was still significantly lighter than initially. Although these patients had a high consumption of staining beverages and food, it was reported not to have an influence on the whitening effect longevity after 6 months. In another study14 where Opalescence PF 10\% was used for 21 days the lightness was maintained after 6 months but tooth sensitivity occurred in $36 \%$ of the subjects. While Swift et al. ${ }^{15}$ stated that patients who used $10 \%$ carbamide peroxide gel nightly for 2 weeks still had a satisfactory aesthetic result 2 years after treatment, some color decrease occurred over time following the original bleaching period. The study by Leonard et al. ${ }^{16}$ revealed that the whitening effect caused by $10 \%$ carbamide peroxide lasted up to 3.8 years in $82 \%$ of the participants and that it is a safe, effective and predictable method of whitening teeth. A retrospective case study ${ }^{2}$ that evaluated safety and participants' perceptions of a night-guard vital bleach, concluded that $10 \%$ carbamide peroxide is safe and effective. Color stability, as perceived by $43 \%$ of the participants, may last 9-12 years post-bleaching.

From the above it became clear that major controversy still exists over the time period necessary until re-bleaching as well as which color changes took place during the bleaching process. Thus, the purpose of this clinical study was to evaluate the effectiveness of Opalescence PF 10\% over a 14- month period.

\section{Materials and methods}

Only students with two sound central maxillary incisors (teeth 11 and 21), in good dental and medical health and not on any medical treatment were selected. Smokers and tetracyclinestained or previously bleached central maxillary incisors were excluded from the study. To remove possible extrinsic stains, a dental prophylaxis was done on the mentioned teeth before 
the start of the bleaching treatment. Seventeen student volunteers aged 19-22 years were instructed to administer an at-home bleach system (Opalescence PF 10\%, carbamide peroxide, potassium nitrate and sodium fluoride; Ultradent Products, Inc., Utah, USA). Customized bleaching trays were made for each patient. The bleach was administered nightly for a 14-day treatment period as described by the manufacturers. Patients were asked to take note of any tooth sensitivity that might occur during the treatment period. A spectrophotometer (Model: CM-26ood, Konica Minolta Sensing, Inc., Japan) set on the CIE L*a*b* color space with a $6 \mathrm{~mm}$ diameter probe was used to determine the tooth shades. Before use, the instrument was calibrated as outlined by the manufacturers (Model: CM-26ood, Konica Minolta Sensing, Inc., Japan). Briefly, a zero calibration into the air was first done, followed by a white calibration on the supplied white calibration plate. Three measurements of one area (6 $\mathrm{mm}$ diameter) at the centre of the crown of the two maxillary central incisors were taken at four different intervals. The intervals being after the prophylaxis (pre-bleach), after 14 days of bleaching (end of the bleaching period), at a 6-month follow-up and at a 14-month follow-up. Seventeen subjects were available for evaluation at the first three intervals, but only 15 subjects were retrievable for the final 14-month assessment. A verbal evaluation system based on a four point descriptor (no sensitivity-0; mild sensitivity-1; moderate sensitivity-2; considerable sensitivity -3 and severe sensitivity -4$)^{18}$ was used to determine the degree of tooth sensitivity after the 14 day bleaching period. The Wilcoxon Signed Rank Sum Test was used to determine the significance of the results.

Full ethical approval for the project was obtained from the Ethical and Research Committee of the University of the Western Cape

\section{Results}

Fig. 1 gives a graph of the 25 percentile, median and 75 percentile differences in the $\mathrm{L}^{*}$ values between the base-line (before treatment) and after treatment (14 days), between base-line and after 6 months as well as between base-line and after 14 months. Fig. 2 shows the same differences in the $\mathrm{a}^{*}$ values, while Fig. 3 gives the differences in the $\mathrm{b}^{*}$ values over time. For all three components $\left(\mathrm{L}^{*}, \mathrm{a}^{*}\right.$ and $\left.\mathrm{b}^{*}\right)$ statistical significant differences $(\mathrm{p}<0.05)$ between base-line values and the values obtained after treatment (14 days later), after 6 months as well as after 14 months were found (Wilcoxon Signed Rank Sum Test).

When comparing the values obtained after treatment to those before treatment-all subjects showed an increase in the $\mathrm{L}^{*}$ value (lighter and brighter). The $\mathrm{a}^{*}$ value decrease amongst 15 of the 17 subjects (less reddish and more greenish), while the $b^{*}$ values also decreased (less yellow) in all the subjects. All the color improvements which occurred between the base-line and 14-day period were found statistically significant $(\mathrm{p}<0.05)$. 


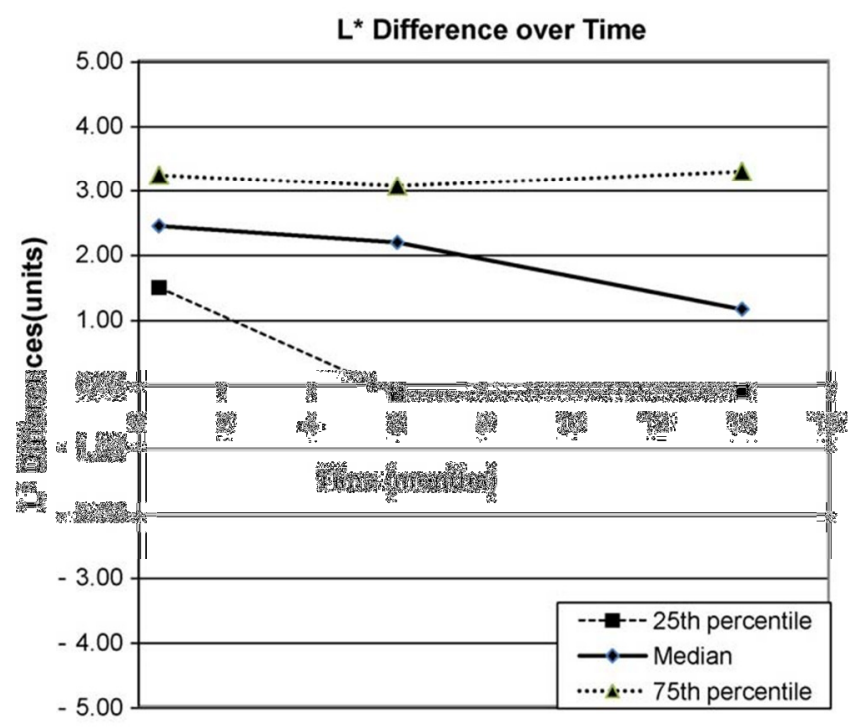

Fig. 1 - The 25 percentile, median and 75 percentile differences in the $L^{*}$ values between the base-line foerore treatment) and after treatmen ( 14 days) after 6 months and afiter 14 months.

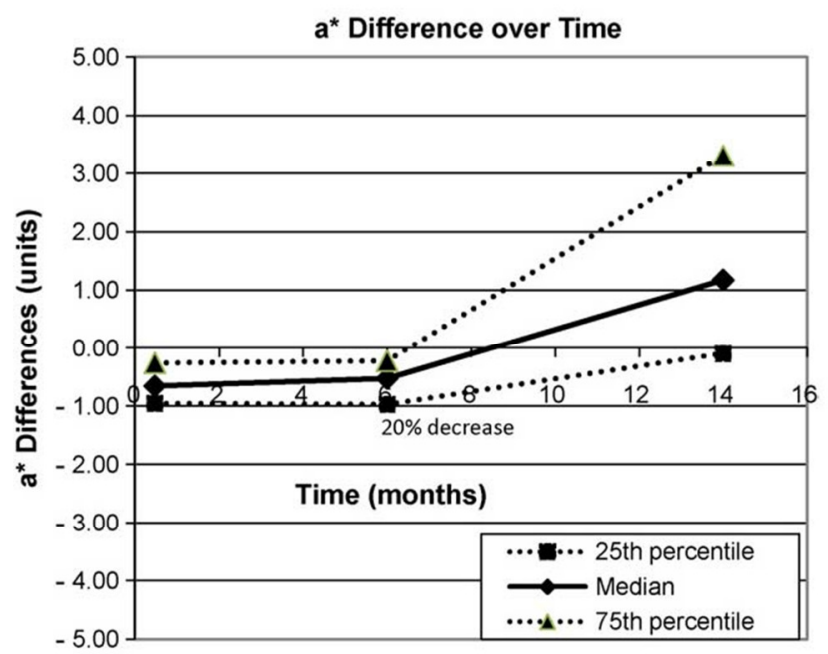

Fig, 2 - The 25 percentile median and 75 percentilo

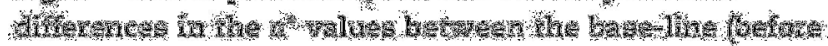
Godthend

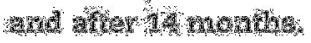




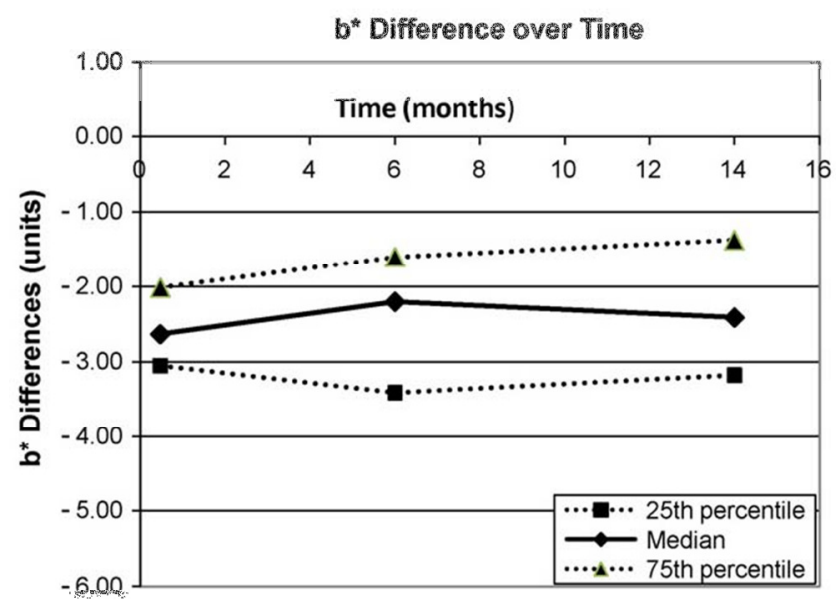

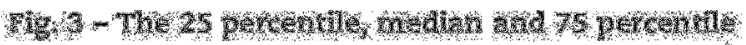

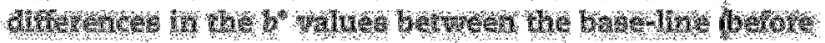

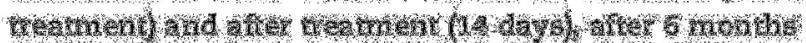
and anter 14 monthas

A comparison between the values obtained before the commencement of bleach and those obtained at 6 months, showed that the teeth still appeared significantly $(\mathrm{p}<0.05)$ more aesthetic 6 months post-bleach than initially. Results gained at the 14-month assessment, indicated that the median values of $\mathrm{L}^{*}$ and $\mathrm{a}^{*}$ decreased further. The $\mathrm{L}^{*}$ value decreased and the $a^{*}$ value increased significantly $(p<0.05)$. However, the median $b^{*}$ value remained more or less stable with an insignificant $(p>0.05)$ change. 


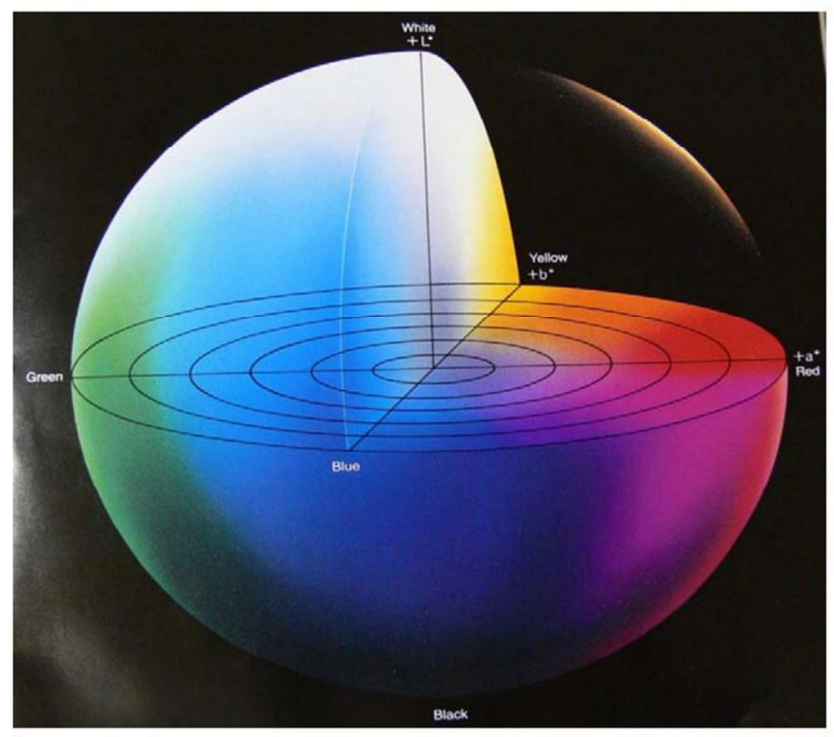

Fig. 4 - Representation of color solid for $L^{*} a^{*} b^{*}$ color space. ${ }^{19}$

The median DEab value was 3.66 after treatment, 3.16 after 6 months and 2.92 after 14 months. Although there was a decrease in the median DEab values (up to 20\% after 14 months), no statistical significant difference ( $p$ > 0.05; Wilcoxon Signed Rank Sum Test) could be demonstrated. Of the 17 subjects evaluated only 4 reported tooth sensitivity on the 4 point rating scale. Two had a rating of 2 and two a rating of 3 . This sensitivity was found to be transient and disappeared at the end of the treatment (14 days).

\section{Discussion}

This clinical study employed the spectrophotometer to determine the color change of teeth up to 14 months post- treatment. The instrument (Model: CM-26ood, Konica Minolta Sensing, Inc., Japan) has diffused illumination with a viewing angle of $8^{\circ}$. The repeatability in terms of the standard deviation when DEab is measured is within 0.04, while the inter instrument agreement for the DEab measurement is within 0.2 (MAV/SCI). The CIE system of color 19(Fig. 4) is a mixture of hue (green, red, blue, yellow, etc.), lightness (bright colors and dark colors) and saturation (vivid colors and dull colors). With the spectrophotometer one can quantify colors by measuring them numerically in a three dimensional color space $\left(\mathrm{L}^{*} \mathrm{a}^{*} \mathrm{~b}^{*}\right)$, where the $\mathrm{L}^{*}$ indicates lightness/darkness (white/black) and $\mathrm{a}^{*}$ and $\mathrm{b}^{*}$ are the cromaticity coordinates. 3 The $\mathrm{a}^{*}$ value varies from a negative side (more greenish) to the positive side (more reddish), while the $\mathrm{b}^{*}$ value varies from the more blue side (negative side) to the more yellow side (positive side)(Fig. 4). The area between the 25 percentile and 50 percentile (Figs. 13 and 5) gives an indication where 50\% of the values are. This spreading of the results can be expected as no selection of subjects were made on the basis of teeth color and it can be expected that the lighter the teeth to be bleached the less the change would be and vice versa. 
Many previous studies used shade guides to measure tooth colors, which have more imitations. With shade guides it is only possible to do one overall measurement of a tooth color. The spectrophotometer has many advantages above the shade guide. Apart from being a small computer it has its own built-in light source, constant illumination/viewing angles and constant "observer", which means observer conditions are uniform for all measurements. The major disadvantage of the shade guide is that it can only measure one value for all the above-mentioned possible variations. It was also reported that evaluation of teeth with a shade guide is unreliable and that intra-evaluator agreement can be as low as $60 \% .20$

Like the shade guide, the color change measurements with the spectrophotometer can also be given by presenting only one value, namely the DE_ab.19, where DE_ab 1/4 $1 / 2$ ðDL_P2 p ðDa_P2 p ðDb_P2_1=2 and $\mathrm{DL}^{*}, \mathrm{Da}^{*}$ and $\mathrm{Db}^{*}$ the changes which occurred in these components. Presenting only the DE_ab results in a masking effect of what really happened in the separate components. In the present study the unit change for the $\mathrm{L}^{*}$ and $\mathrm{b}^{*}$ values are more or less of the same magnitude, about 2.5 (Figs. 1 and 3) but the $\mathrm{a}^{*}$ values are in the order of only about 0.5 . Thus, had only the $\mathrm{DE}_{-}$ab been measured the real color change for the $\mathrm{a}^{*}$ value would not have been visible. Others $13,21-23$ also reported that tooth whitening mainly occurs in the $\mathrm{L}^{*}$ and $\mathrm{b}^{*}$ values. The $\mathrm{DE} \_$ab values (Fig. 4) also show a slow decrease over the 14-month period. it shows a $13.7 \%$ decrease after 6 months and a $20.2 \%$ decrease after 14 months. However, it can be seen that this slow decrease is mainly supported by the $b$ value after a 14-month period (Figs. 1-3) which then gives a wrong impression when studying only the $\mathrm{DE}_{-}$ab values. The $\mathrm{DE} \_\mathrm{ab}$ increase of 3.66 units is not toofar from the 3 units reported (dos Santos 2008). However, a 5.35 unit increase was also reported.24 In contrast to this study, many studies were done but most did not apply the manufacturer's recommendation for the treatment period (over night for 14 days). It also seems that the treatment period has an effect on the results and that a longer period of treatment and stronger peroxide solution is associated with better whitening. ${ }^{13,14,17,24}$

From Fig. 1 it can be seen that the $L^{*}$ values (whiteness/ brightness) decreased with time but were still, after three time periods (14 days, 6 months and 14 months), significantly better than at base-line. After 6 months the decrease was only 10.6\% (Fig. 1), but after 14 months the decrease in the $\mathrm{L}^{*}$ value was $52.4 \%$ of the value just after treatment (14 days). The question however is whether this decrease is still acceptable as far as the whiteness/brightness of teeth are concerned or is it already necessary to re-bleach. The answer to this question might vary from person to person A decrease of $52.4 \%$ could be considered an indication that re-bleaching is overdue.

If we look at the $\mathrm{a}^{*}$ values (green/red) over time it seems that after about 8 months of treatment there was no difference from the original base-line value i.e. where the line of the graph (Fig. 2) intersects the y-axis at $a$ value of o. However, the figure even showed a further 
decrease (more reddish) than at the base-line value after 14 months. This means that although there was initially a decrease in the color red (more negative) there was an increase of the red color after 6 months and a further increase in the red color to a value more reddish than that at the base-line. Deleted sentence Ritter et al. ${ }^{17}$ stated that a $10 \%$ carbamide peroxide solution provided patients with aesthetic results up to 12 years post- bleach. It should however be taken into consideration that those results were only based on patients' perceptions (and only $43 \%$ of the initial sample size) and not on valuable parameters like a spectrophotometer or even a shade guide. Although Swift et al. ${ }^{15}$ concluded that $10 \%$ carbamide peroxide still had satisfactory results 2 years post-bleaching, it was also measured with tooth shade guides.

In a recent study ${ }^{13}$ on a $10 \%$ carbamide peroxide it was reported that the whitening effect remained similar after a 6- month period, although the treatment period was $2 \mathrm{~h} /$ day for 3 weeks. Goo et al. ${ }^{24}$ also found $10 \%$ carbamide peroxide effective with a linear increase in the whiteness as the period increased. Deliperi and Bardwell4 reported no decrease of the shade after a 2-year follow-up with $10 \%$ carbamide peroxide.

Tooth sensitivity and gingival irritation is normally a common problem in tooth bleaching. However, in this study with Opalescence PF 10\% it was found to be light and in the low range (less than $23 \%$ of our subjects). It is reported that even different brands of $10 \%$ carbamide peroxide bleaching agents have different levels of penetration and may also have different levels of sensitivity or bleaching efficacies. 5 In other clinical studies, Opalescence PF 10\% was also shown to have low sensitivity.13,14,26 This could be due to the presence of fluoride and potassium nitrate. It is also reported that the urea present in Opalescence is a scavenger for residual hydrogen peroxide and hydroxyl radicals which might also have an influence on the sensitivity. ${ }^{6}$ Furthermore, hydrogen peroxide in the presence of $\mathrm{NaF}$ was found to stimulate the formation of fluoridated hydroxyapatite (FHAP) on the hydro- xyapatite (HAP), while the formation of $\mathrm{CaF}_{2}$ was accelerated with the dissolution of the HAP. ${ }^{28,29}$ This could also reduce the sensitivity, while tooth sensitivity with $10 \%$ carbamide peroxide was reported to be none or mild. ${ }^{13}$

\section{Conclusions}

Opalescence $\mathrm{PF} 10 \%$ is an effective at-home bleaching product and can be used by patients with minimal side-effects. It does provide significant aesthetic results in all 3 color coordinates $\left(\mathrm{L}^{*}, \mathrm{a}^{*}\right.$ and $\left.\mathrm{b}^{*}\right)$ for up to 6 months post-bleach. Fourteen months after tooth whitening, the $b^{*}$ value (which indicate yellowness) remained more or less stable, even though the $L^{*}$ and $a^{*}$ values had decreased significantly. It is suggested that re-bleaching after 6 months is not necessary but should be done at about 14 months post bleaching. 


\section{R E F E R E N C E S}

1.Joiner A. The bleaching of teeth: a review of the literature. Journal of Dentistry 2006;34:412-9.

2. Heymann HO. Tooth whitening: facts and fallacies. British Dental Journal 2005;198:514.

3. Cavalli V, Arrais CAG, Giannini M, Ambrosano GMB. High- concentrated carbamide peroxide bleaching agents effects on enamel surface. Journal of Oral Rehabilitation 2004;31:155-9.

4. Zantner C, Beheim-Schwarzbach N, Neumann K, Kielbassa AM. Surface microhardness of enamel after different home bleaching procedures. Dental Materials 2007;23:243-50.

5. Basting RT, Rodrigues Jr AL, Serra MC. The effect of seven carbamide peroxide bleaching agents on enamel microhardness over time. Journal of the American Dental Association 2003;134:1335-42.

6. Auschill TM, Hellwig E, Schmidale S, Sculean A, Arweiler NB. Efficacy, side-effects and patients' acceptance of different bleaching techniques (OTC, in-office, at-home). Operative Dentistry 2005;30:156-63.

7. Basting RT, Rodrigues Jr AL, Serra MC. The effect of $10 \%$ carbamide peroxide bleaching material on microhardness of sound and demineralized enamel and dentine in situ. Operative Dentistry 2001;26:531-9.

8. de la Pena VA, Cabrita OB. Comparison of the clinical efficacy and safety of carbamide peroxide and hydrogen peroxide in at-home bleaching gels. Quintessence International 2006;37:551-6.

9. Haywood VB, Heyman HO. Nightguard vital bleaching: how safe is it? Quintessence International 1991;22:515-23.

10. Haywood VB. History, safety and effectiveness of current bleaching techniques and applications of the nightguard vital bleaching technique. Quintessence International 1992;23:471-88.

11.Pugh G, Zaidel L, Lin N, Stranick M, Bagley D. High levels of hydrogen peroxide in overnight tooth-whitening formulas: effects on enamel and pulp. Journal of Esthetic and Restorative Dentistry 2005;17:40-5.

12. Majeed A, Grobler SR, Moola MH, Rossouw RJ, van Wyk Kotze TJ. Effect of four different Opalescence tooth- whitening products on enamel microhardness. South African Dental Journal 2008;63:282-6.

13. Meireles SS, Heckmann SS, Santos IS, Della Bona A, Demarco FF. A double blind randomized clinical trial of at- home tooth bleaching using two carbamide peroxide concentrations: 6-month follow-up. Journal of Dentistry 2008;36:878-84.

14. dos Santos Medeiros MC, de Lima KC. Effectiveness of nightguard vital bleaching with 10\% carbamide peroxide-a clinical study. Journal of the Canadian Dental Association 2008;74:163-1163.

15. Swift Jr EJ, MayJr KN, Wilder Jr AD, Heymann HO, Bayne SC. Two-year clinical evaluation of tooth whitening using an at- home bleaching system. Journal of Esthetic Dentistry 1999;11:36-42. 
16. Leonard Jr RH, Bentley C, Eagle JC, Garland GE, Knight MC, Phillips C. Nightguard vital bleaching: a long-term study on efficacy, shade retention, side effects, and patient's perceptions. Journal of Esthetic and Restorative Dentistry 2001;13:357-69.

17. Ritter AV, Leonard Jr RH, St Georges AJ, Caplan DJ, Haywood VB. Safety and stability of nightguard vital bleaching: 9-12 years post-treatment. Journal of Esthetic and Restorative Dentistry 2002;14:275-85.

18. Mokhlis GR, Matis BA, Cochran MA, Eckert GJ. A clinical evaluation of carbamide peroxide and hydrogen peroxide whitening agents during daytime use. Journal of the American Dental Association 2000;131:1269-77.

19. Minolta, Precise color communication, Minolta, Co., Ltd., Osaka, Japan, 1994; 9242-4830-92 IHCAJ.

20. Horn D, Bulan-Brady J, Hicks M. Sphere spectrophotometer versus human evaluation of tooth shade. Journal of Endodontics 1998;24:786-90.

21. American Dental Association. ADA Acceptar Program Guidelines Dentist dispensed home-use tooth bleaching products ADA Council on Scientific Affairs; 2006. http:// www.ada.org/ada/seal/standards/guide home bleach.pdf [Retrieved on May 2009].

22. Luo W, Westland S, Brunton P, Ellwood R, Pretty IA, Mohan N. Comparison of the ability of different color indices to assess changes in tooth whiteness. Journal of Dentistry 2007;35:109-16.

23. Ishikawa-Nagai S, Terui T, Ishibasi K, Weber HP, Ferguson M. Comparison of effectiveness of two 10\% carbamide peroxide tooth-bleaching systems using spectrophotometric measurements. Journal of Esthetic and Restorative Dentistry 2004;16:368-75.

24. Goo DH, Kwon TY, Nam SH, Kim HJ, Kim KH, Kim YJ. The efficiency of $10 \%$ carbamide peroxide gel on dental enamel. Dental Materials Journal 2004;23:522-7.

25. Deliperi S, Bardwell DN. Two-year clinical evaluation of nonvital tooth whitening and resin composite restorations. Journal of Esthetic and Restorative Dentistry 2005;17:369-78.

26. Ziebolz D, Helms K, Hannig C, Attin T. Efficacy and oral side effects of two highly concentrated tray-based bleaching systems. Clinical Oral Investigations 2007;11:267-75.

27. Farmer DS, Burcham P, Marin PD. The ability of thiourea to scavenge hydrogen peroxide and hydroxyl radicals during the intra-coronal bleaching of bloodstained root-filled teeth. Australian Dental Journal 2006;51:146-52.

28. Tanizawa Y. Reaction characteristics of a tooth-bleaching agent containing $\mathrm{H}_{2} \mathrm{O} 2$ and NaF. Operative Dentistry 2005;30:265-70.

29. Tanizawa A. Reaction characteristics of a tooth-bleaching agent containing $\mathrm{H}_{2} \mathrm{O} 2$ and NaF: in vitro study of crystal structure change in treated hydroxyapatite and chemical states of incorporated fluorine. Journal of Cosmetic Science 2005;56:121-34. 